United Nations Educational Scientific and Cultural Organization and

International Atomic Energy Agency

THE ABDUS SALAM INTERNATIONAL CENTRE FOR THEORETICAL PHYSICS

\title{
RANDOM FIXED POINTS OF SET-VALUED MAPS
}

\author{
Naseer Shahzad \\ Department of Mathematics, Quaid-i-Azam University, \\ Islamabad, Pakistan \\ and
}

The Abdus Salam International Centre for Theoretical Physics, Trieste, Italy.

\begin{abstract}
Some random fixed point theorems for set-valued random operators under very mild conditions are established. Some recent results of O'Regan ([Proc. Amer. Math. Soc. 126 (1998), 3045-3053] and [Computers Math. Applic. 35 (1998), 27-34]) are improved significantly. The discussion in this paper underlines, in addition to generality, the unifying aspects of our result.
\end{abstract}

MIRAMARE - TRIESTE

August 1998 


\section{INTRODUCTION}

During the last twenty years many results concerning random fixed points of various types of operators have been obtained, and a number of their applications have been given. Hence, it is necessary to prove a random fixed point theorem under very mild conditions that includes most of the known results and which is applicable to random differential equations, random integral equations, random approximations etc. In this paper, we achieve this goal.

Let $(\Omega, \Sigma)$ be a measurable space, $S$ a complete subset of a metric space $X$, and $C(X)$ the family of all non-empty closed subsets of $X$. The object of this paper is two-fold.

First, we prove that if $T: \Omega \times S \longrightarrow C(X)$ is a continuous random operator which satisfies the condition $(A)$, then $T$ has a random fixed point. This result unifies and extends a number of known, as well as some new, random fixed point theorems. Our result includes, as special cases, the earlier theorem of Itoh [4] as well as the recent results of Benavides, Acedo and Xu [2], O’Regan [10, 11], Xu [16], and Tan and Yuan [14].

More recently, O'Regan [9] obtained a random fixed point theorem for a continuous hemicompact 1-set-contractive random operator $T: \Omega \times S \rightarrow C K(X)$ which satisfies the following conditions:

1. If $\left\{x_{n}\right\} \subset S$ and $y_{n} \in T\left(\omega, x_{n}\right)$ for all $n$ are such that $x_{n}-y_{n} \longrightarrow 0$ as $n \longrightarrow \infty$, then there exists $x \in S$ with $x \in T(\omega, x)$.

2. If $\left\{\left(x_{j}, \lambda_{j}\right)\right\}_{j=1}^{\infty}$ is a sequence in $\partial S \times[0,1]$ converging to $(x, \lambda)$ with $x \in \lambda T(\omega, x)$ and $0 \leq \lambda<1$, then there exists $j_{0} \in\{1,2, \ldots\}$ with $\left\{\lambda_{j} T\left(\omega, x_{j}\right)\right\} \subset S$ for each $j \geq j_{0}$.

Here $C K(X)$ denotes the family of all non-empty compact convex subsets of $X$ and $\partial S$ the boundary of $S$. In this context, we apply our main theorem to the derivation of a random fixed point theorem which improves O'Regan's result significantly. We also observe that the hemicompactness of $T$ implies condition (1) and that O'Regan's theorem remains valid if condition (1) is dropped.

We remark specifically that our result may be employed to obtain several random fixed point theorems for various classes of random maps, that have recently been studied by a number of authors, which are 1-set-contractive (e.g., Lin [6], Liu [7], Shahzad [12], Shahzad and Latif [13], Tan and Yuan [15], Yuan and Yu [17] etc.). This indicates the generality and the unifying aspect of our result.

\section{Preliminaries}

Let $(\Omega, \Sigma)$ be a measurable space and $S$ a non-empty subset of a metric space $X$. We denote by $2^{S}$ the family of all subsets of $S$ and by $C B(S)$ the family of all non-empty closed bounded subsets of $S$. A mapping $G: \Omega \longrightarrow 2^{S} \backslash\{\phi\}$ is called measurable if, for each open subset $U$ of $S, G^{-1}(U)=\{\omega \in \Omega: G(\omega) \cap U \neq \phi\} \in \Sigma$. A mapping $\xi: \Omega \longrightarrow S$ is said to be a measurable selector of measurable mapping $G: \Omega \longrightarrow 2^{S} \backslash\{\phi\}$ if $\xi$ is measurable and $\xi(\omega) \in G(\omega)$ for 
each $\omega \in \Omega$. A mapping $T: \Omega \times S \longrightarrow 2^{X} \backslash\{\phi\}$ is called a random operator if, for any fixed $x \in S$, the map $T(., x): \Omega \longrightarrow 2^{X} \backslash\{\phi\}$ is measurable. A mapping $\xi: \Omega \longrightarrow S$ is said to be a random fixed point of a random operator $T: \Omega \times S \longrightarrow 2^{X} \backslash\{\phi\}$ if $\xi(\omega) \in T(\omega, \xi(\omega))$ for each $\omega \in \Omega$. Let $X$ be a Banach space and $A$ a bounded subset of $X$. The Kuratowski measure of non-compactness is defined by $\alpha(A)=\inf \{c>0: A$ can be covered by a finite number of sets of diameter $\leq c\}$. The Hausdorff measure of non-compactness is defined by $\chi(A)=\inf \{c>0: A$ can be covered by a finite number of open balls of radius $\leq c\}$. A mapping $T: S \longrightarrow C(X)$ is called (1) $k$-set-contractive (w.r.t. $\alpha$ ) if $T(S)$ is bounded and, for each bounded subset $A$ of $S, \alpha(T(A)) \leq k \alpha(A)$, where $k \geq 0 ;(2)$ condensing (w.r.t. $\alpha$ ) if $T(S)$ is bounded and, for each bounded subset $A$ of $S$ with $\alpha(A)>0, \alpha(T(A))<\alpha(A)$. As in the case of $\alpha$, corresponding to $\chi$ we have $k$-set contractive (w.r.t. $\chi$ ) and condensing (w.r.t. $\chi$ ) maps. A mapping $T: S \longrightarrow C(X)$ is said to satisfy the condition $(A)$ if for any sequence $\left\{x_{n}\right\}$ in $S$ and $D \in C(S)$ such that $d\left(x_{n}, D\right) \longrightarrow 0$ and $d\left(x_{n}, T\left(x_{n}\right)\right) \longrightarrow 0$ as $n \longrightarrow \infty$, there exists $x_{0} \in D$ with $x_{0} \in T\left(x_{0}\right)$, where $d(x, D)=\inf \{d(x, y): y \in D\}$. A map $T: S \longrightarrow C(X)$ is called hemicompact if each sequence $\left\{x_{n}\right\}$ in $S$ has a convergent subsequence whenever $d\left(x_{n}, T\left(x_{n}\right)\right) \longrightarrow 0$ as $n \longrightarrow \infty$. We observe that every continuous hemicompact map satisfies the condition $(A)$. Also the condition $(A)$ is always true for continuous condensing mappings. A random operator $T: \Omega \times S \longrightarrow C(X)$ is said to be continuous ( $k$-set-contractive, condensing, etc.) if the map $T(\omega,):. S \longrightarrow C(X)$ is so for each $\omega \in \Omega$.

\section{MAIN RESULTS}

Using a variant of the method first employed by Itoh [4](see also Beg and Shahzad [1], Benavides, Acedo and Xu [2], Tan and Yuan [14]), we prove the following theorem which incorporates some recent results (see, for examples, Beg and Shahzad [1], Benavides, Acedo and Xu[2, Theorem 3.3 and Corollary 3.3], Itoh[4, Theorem 3.1], Tan and Yuan[14, Theorem 2.3], etc.).

Theorem 3.1. Let $S$ be a non-empty separable complete subset of a metric space $X$ and $T$ : $\Omega \times S \longrightarrow C(X)$ a continuous random operator satisfying the condition $(A)$. Then $T$ has a deterministic fixed point if and only if $T$ has a random fixed point.

Proof. It is enough to show that if $T$ has a deterministic fixed point, then $T$ has a random fixed point. For each $\omega \in \Omega$, set $G(\omega)=\{x \in S: x \in T(\omega, x)\}$. By assumption, $G(\omega)$ is non-empty and complete for each $\omega \in \Omega$. We now prove that the mapping $G: \Omega \longrightarrow 2^{S} \backslash\{\phi\}$ is measurable. For any non-empty closed subset $D$ of $S$, let

$$
L(D)=\bigcap_{n=1}^{\infty} \bigcup_{i=1}^{\infty}\left\{\omega \in \Omega: d\left(x_{i}, T\left(\omega, x_{i}\right)\right)<\frac{2}{n}\right\},
$$

where $\left\{x_{i}\right\}$ is a countable dense subset of $D$ and $d(x, D)=\inf \{d(x, y): y \in D\}$. As in the proof of Tan and Yuan[14, Theorem 2.3], $G^{-1}(D) \subset L(D)$. On the other hand, if $\omega \in L(D)$, then, for each $n$, there exists $i(n)$ such that $d\left(x_{i(n)}, T\left(\omega, x_{i(n)}\right)\right)<\frac{2}{n}$. Since $T$ satisfies the condition 
$(A)$ and $d\left(x_{i(n)}, T\left(\omega, x_{i(n)}\right)\right) \longrightarrow 0$, it follows that there exists some $x_{0} \in D$ with $x_{0} \in T\left(\omega, x_{0}\right)$. Hence $G(\omega) \cap D \neq \phi$ and so $\omega \in G^{-1}(D)$. Therefore, $G^{-1}(D)=L(D)$ and $G$ is measurable. Now, by the Kuratowski and Ryll-Nardzewski selection theorem [5], $G$ has a measurable selector $\xi: \Omega \longrightarrow S$. This $\xi$ is clearly a random fixed point of $T$.

Corollary 3.2. [14, Theorem 2.8]. Let $S$ be a non-empty separable complete subset of a metric space $X$ and $T: \Omega \times S \longrightarrow C(X)$ a continuous hemicompact random operator. Then $T$ has a deterministic fixed point if and only if $T$ has a random fixed point.

Proof. Since every continuous hemicompact map satisfies the condition $(A)$, the corollary follows from Theorem 2.1.

Corollary 3.3. [2, Theorem 3.3]. Let $S$ be a non-empty separable closed convex subset of a Banach space $X$ and $T: \Omega \times S \longrightarrow C B(X)$ a continuous condensing random operator (w.r.t. $\alpha)$. Suppose $T$ has a deterministic fixed point. Then $T$ has a random fixed point.

Proof. Since every continuous condensing map satisfies the condition $(A)$, the corollary follows immediately from Theorem 2.1.

Now we assume that the interior of $S$ may be empty. This case is very useful in studying ordinary differential equations on noncompact intervals [3, 8]. Thus the following result may be applied to establish the existence principle for random differential equations on noncompact intervals.

Theorem 3.4. Let $S$ be a non-empty separable closed convex subset of a Hilbert space $X$ with $0 \in S$ and $T: \Omega \times S \longrightarrow C K(X)$ a continuous 1-set-contractive random operator (w.r.t. $\alpha$ or $\chi)$ satisfying the condition (A). If (2) holds, then T has a random fixed point.

Proof. For each $n=2,3, \ldots$, define a mapping $T_{n}: \Omega \times S \longrightarrow C K(X)$ by $T_{n}(\omega, x)=(1-$ $\left.\frac{1}{n}\right) T(\omega, x)$. Then $T_{n}$ is a condensing random operator (w.r.t. $\alpha$ or $\chi$ ). For each $\omega \in \Omega$, let $\left\{\left(x_{j}, \lambda_{j}\right)\right\}_{j=1}^{\infty}$ be a sequence in $\partial S \times[0,1]$ and $\left(x_{j}, \lambda_{j}\right) \longrightarrow(x, \lambda)$ as $j \longrightarrow \infty$ with $x \in \lambda T_{n}(\omega, x)$ and $0 \leq \lambda<1$. Then, for any $\omega \in \Omega, \lambda_{j} T_{n}\left(\omega, x_{j}\right)=\lambda_{j}\left(1-\frac{1}{n}\right) T\left(\omega, x_{j}\right)=\mu_{j} T\left(\omega, x_{j}\right) \subset S$, for $j$ sufficiently large. It is clear that $\mu_{j}=\lambda_{j}\left(1-\frac{1}{n}\right)$ is a sequence in $[0,1]$ with $\mu_{j} \longrightarrow \lambda\left(1-\frac{1}{n}\right)=$ $\mu$ (say), $0 \leq \mu<1$, and $x \in \lambda T_{n}(\omega, x)=\lambda\left(1-\frac{1}{n}\right) T(\omega, x)=\mu T(\omega, x)$ for each $\omega \in \Omega$. Hence, by $\left[9\right.$, Theorem 2.1], $T_{n}(\omega,$.$) has a fixed point x_{n}$ (depending on $\omega$ ), that is, $x_{n} \in\left(1-\frac{1}{n}\right) T\left(\omega, x_{n}\right)$ for each $\omega \in \Omega$. Choose $y_{n} \in T\left(\omega, x_{n}\right)$ with $x_{n}=\left(1-\frac{1}{n}\right) y_{n}$. Now, $x_{n}-y_{n}=\left(-\frac{1}{n}\right) y_{n} \rightarrow 0$ as $n \longrightarrow \infty$. Further, $d\left(x_{n}, T\left(\omega, x_{n}\right)\right) \leq\left\|x_{n}-y_{n}\right\|=\left\|\left(-\frac{1}{n}\right) y_{n}\right\| \longrightarrow 0$ as $n \longrightarrow \infty$. It follows, by the condition $(A)$, that there exists an $x \in S$ such that $x \in T(\omega, x)$ for each $\omega \in \Omega$. An application of Theorem 3.1 yields that $T$ has a random fixed point. 
Corollary 3.5. [9, Theorem 3.1]. Let $S$ be a non-empty separable closed convex subset of a Hilbert space $X$ with $0 \in S$ and $T: \Omega \times S \longrightarrow C K(X)$ a continuous hemicompact 1-setcontractive random operator (w.r.t. $\alpha$ or $\chi$ ). Suppose (1) and (2) are satisfied. Then T has a random fixed point.

Corollary 3.6. [9, Theorem 3.2]. Let $S$ be a non-empty separable closed convex subset of a Hilbert space $X$ with $0 \in S$ and $T: \Omega \times S \longrightarrow C K(X)$ a continuous condensing random operator (w.r.t. $\alpha$ ). Suppose (2) holds. Then $T$ has a random fixed point.

\section{Remark 3.7.}

1. Theorem 3.1 is a significant generalization of most of the results that have appeared on random fixed points. In particular, the more recent results of O'Regan (Theorems 2.1 to 2.3[10] and Theorem 3.1[11]) can be viewed as special cases of Theorem 3.1. It is interesting to compare the hemicompactness of $T$ to the property (B) of Theorem 3.1[11]. We further add that the hemicompactness of $T$ may be dropped.

2. In view of Theorem 3.4, we observe that Corollary 3.5[9, Theorem 3.1] remains valid without the assumption of condition (1).

3. We also note that the hemicompactness of $T$ implies condition (1) in Corollary $3.5[9$, Theorem 3.2]. Indeed, fix $\omega \in \Omega$ arbitrarily. Assume $T$ is hemicompact. Let $\left\{x_{n}\right\} \subset S$ and $y_{n} \in T\left(\omega, x_{n}\right)$ for all $n$ be such that $x_{n}-y_{n} \longrightarrow 0$ as $n \longrightarrow \infty$. Then $d\left(x_{n}, T\left(\omega, x_{n}\right)\right) \leq$ $\left\|x_{n}-y_{n}\right\| \longrightarrow 0$ as $n \longrightarrow \infty$. It follows, by the hemicompactness of $T$, that there exists a subsequence $\left\{x_{m}\right\}$ of $\left\{x_{n}\right\}$ such that $x_{m} \longrightarrow x_{0} \in S$. The continuity of $T$ further implies that $x \in T(\omega, x)$. Hence $T$ satisfies condition (1).

4. Condition (1) is always true for an operator $T: \Omega \times S \longrightarrow C(X)$ that satisfies the condition $(A)$.

Problem. It seems to be an interesting problem to prove Theorem 3.1 for a continuous random operator $T: \Omega \times S \longrightarrow C(X)$ satisfying conditon (1). This may provide an anwser to an open question, whether the (deterministic) fixed point property for nonexpansive mappings implies the random fixed point property for (random) nonexpansive mappings (cf. [16]). It is well known that if $X$ is uniformly convex and $S$ is closed bounded convex, then condition (1) holds for every nonexpansive mapping $T: S \longrightarrow X$. For details, we refer to $[2,16]$.

Acknowledgments. The author would like to express his gratitude to the Abdus Salam International Centre for Theoretical Physics, Trieste, Italy and the Commission on Development and Exchange of the International Mathematical Union for financial support. This work was done while the author was visiting the Abdus Salam ICTP, Trieste, Italy. 


\section{References}

[1] I. Beg and N. Shahzad, A general fixed point theorem for a class of continuous random operators, New Zealand J. Math. 26 (1997), 21-24.

[2] T. D. Benavides, G. L. Acedo and H. K. Xu, Random fixed points of set-valued operators, Proc. Amer. Math. Soc. 124 (1996), 831-838.

[3] M. Furi and P. Pera, A continuation method on locally convex spaces and applications to ordinary differential equations on noncompact intervals, Ann. Polon. Math. 47 (1987), 331-346.

[4] S. Itoh, Random fixed point theorems with an application to random differential equations in Banach spaces, J. Math. Anal. Appl. 67 (1979), 261-273.

[5] K. Kuratowski and C. Ryll-Nardzewski, A general theorem on selectors, Bull. Acad. Polon. Sci. Ser. Sci. Math. Astronom. Phys., 13 (1965), 397-403.

[6] T. C. Lin, Random approximations and random fixed point theorems for continuous 1-set-contractive random maps, Proc. Amer. Math. Soc. 123 (1995), 1167-1176.

[7] L. S. Liu, Some random approximations and random fixed point theorems for 1-set-contractive random operators, Proc. Amer. Math. Soc. 125 (1997), 515-521.

[8] D. O'Regan, Some fixed point theorems for concentrative mappings between locally convex linear topological spaces, Nonlinear Anal. 27 (1996), 1436-1446.

[9] D. O'Regan, New fixed-points results for 1-set-contractive set valued maps, Computers Math. Applic. 35 (1998), 27-34

[10] D. O'Regan, A continuation type result for random operators, Proc. Amer. Math. Soc. 126 (1998), $1963-1971$.

[11] D. O'Regan, Fixed points and random fixed points for weakly inward approximable maps, Proc. Amer. Math. Soc. 126 (1998), 3045-3053.

[12] N. Shahzad, Random fixed point theorems for various classes of 1-set-contractive maps in Banach spaces, $J$. Math. Anal. Appl. 203 (1996), 712-718.

[13] N. Shahzad and S. Latif, On random approximations and random fixed point theorems for 1-set-contractive maps, The AS-ICTP Preprint IC/98/90, Trieste, 1998.

[14] K. K. Tan and X. Z. Yuan, Random fixed point theorems and approximation in cones, J. Math. Anal. Appl. 185 (1994), 378-390.

[15] K. K. Tan and X. Z. Yuan, Random fixed point theorems and approximation, Stoch. Anal. Appl. 15 (1997), 103-123.

[16] H. K. Xu, Some random fixed point theorems for condensing and nonexpansive operators, Proc. Amer. Math. Soc. 110 (1990), 395-400.

[17] X. Z. Yuan and J. Yu, Random fixed point theorems for nonself mappings, Nonlinear Anal. 26 (1996), 1097-1102. 